\title{
Carcinoma of the Ampulla of Vater Associated With or Without Adenoma: A Clinicopathologic Analysis of 198 Cases With Reference to p53 and Ki-67 Immunohistochemical Expressions
}

Masaki Takashima, M.D., Takashi Ueki, M.D., Eishi Nagai, M.D., Takashi Yao, M.D., Koji Yamaguchi, M.D., Masao Tanaka, M.D., Masazumi Tsuneyoshi, M.D.

Department of Anatomic Pathology (MT, TU, EN, TY, MT), Pathological Sciences, Graduate School of Medical Sciences, Kyushu University; and Department of Surgery and Oncology (KY, MT), Medicine and Surgery, Graduate School of Medical Sciences, Kyushu University, Fukuoka, Japan. Takashi Ueki and Eishi Nagai currently belong to Department of Surgery and Oncology, Medicine and Surgery, Graduate School of Medical Sciences, Kyushu University, Fukuoka, Japan.

Adenomatous areas are found frequently within or in the vicinity of carcinoma of the ampulla of Vater. This makes definite diagnosis difficult in the preoperative examination. The adenoma-carcinoma development hypothesis is generally accepted for colorectal tumors. Recently, a genetic alteration model during colorectal tumor development has attracted much attention, leading to various studies. We studied clinicopathologic features, prognostic factors, and the alteration of the p53 tumor suppressor gene using p53 immunohistochemical staining in pure adenomas, pure carcinomas, and carcinomas with adenomatous areas. A proliferative activity of the tumors using Ki-67 was also evaluated. Nine cases of pure adenoma and 198 cases of carcinoma of the ampulla of Vater were selected for this study. Among the 198 cases of the carcinoma, 83 cases $(42 \%)$ had adenomatous areas. Positivity of p53 immunohistochemical staining was $0 \%$ in pure adenomas, $36 \%$ in the adenomatous areas of carcinomas with adenomatous areas and $62 \%$ in the carcinomatous areas of carcinomas with adenomatous areas, and $56 \%$ in pure carcinoma. Accumulation of p53 protein and the Ki-67 labeling index revealed no significant difference in prognosis. The clinicopathological factors examined were as follows: degree of invasion of the surrounding tissue, such as duodenal wall; pancreatic parenchyma; the

Copyright (C) 2000 by The United States and Canadian Academy of Pathology, Inc.

VOL. 13, NO. 12, P. 1300, 2000 Printed in the U.S.A.

Date of acceptance:

Address reprint requests to: Professor Masazumi Tsuneyoshi, Department of Anatomic Pathology, Pathological Sciences, Graduate School of Medical Sciences, Kyushu University, 3-1-1 Maidashi, Higashi-ku, Fukuoka 812-8582, Japan; e-mail: masazumi@surgpath.med.kyushu-u.ac.jp; fax: 011810926425968. presence or absence of lymphatic permeation; venous invasion; perineural invasion; the presence of regional lymph node metastasis; and TNM stage. Each of the clinicopathological factors showed a significant difference. Multivariate analysis revealed strong predictors for a worse prognosis: presence of lymphatic permeation, invasion of the pancreas, and perineural invasion. In conclusion, our results are consistent with the adenoma-carcinoma development hypothesis. It would seem that the molecular events leading to p53 accumulation in neoplasms of the ampulla of Vater occur relatively late during the oncogenetic process. Moreover, we think it may be useful to refer to the p53 overexpression in the diagnosis of ampullary tumors.

KEY WORDS: Adenoma-carcinoma development hypothesis, Adenomatous area, Ampullary carcinoma, Early carcinoma, p53.

Mod Pathol 2000;13(12):1300-1307

Carcinoma of the ampulla of Vater has a relatively good prognosis when compared with the case of other biliary tract neoplasms such as pancreatic carcinoma or bile duct carcinoma; nevertheless, its 5 -year survival rate ranges from 6 to $61 \%(1-5)$. Carcinoma of the ampulla of Vater can be detected earlier than other biliary tract neoplasms because it involves symptoms such as jaundice or obstructive inflammation that are more easily recognizable. However, cases of carcinoma of the ampulla of Vater extending beyond the muscle of Oddi have shown poor prognosis in studies elsewhere $(2,5)$. There have been statistically significant differences in the survival curves between cases confined within the muscle of Oddi and cases showing further extension, indicating early carcinoma and ad- 
vanced carcinoma, respectively $(2,5)$. These tumors often contain adenomatous areas composed of mild to moderate dysplastic epithelium (6-10). Within such limited areas, it is difficult to diagnose histologically whether a tumor of the ampulla of Vater is in fact benign or malignant.

A genetic alteration model of colorectal tumor development as proposed by Vogelstein et al. has attracted much interest, leading to several studies (11, 12). They considered that the accumulation of alterations affecting at least one dominantly acting oncogene and several tumor suppressor genes is responsible for the development of colorectal tumors. Mutation of the p53 tumor suppressor gene is the most common genetic alteration in human malignancies and is considered to be one of the most important genetic alterations in the model. The overexpression of p53 protein has been widely reported in various neoplasms, including the periampullary region (13-18). Monoclonal antibody Ki-67, which detects a nuclear antigen expressed in all the phases of the proliferating cell cycle except for G0, has become a reliable means of accurately assessing the growth fraction of human neoplasms (19-22).

In this study, we compared the different clinicopathological findings of pure adenomas, early and advanced ampullary carcinomas, and adenomatous areas within these lesions, with reference to the accumulation of p53 protein and the Ki-67 labeling index. Our aim was to investigate the usefulness of immunohistochemical staining with p53 protein or Ki-67 monoclonal antibody in the diagnosis of carcinoma of the ampulla of Vater and to evaluate those clinicopathological factors that were considered to predict prognosis precisely. Moreover, we intended to evaluate whether the adenoma-carcinoma development hypothesis held true.

\section{MATERIALS AND METHODS}

During the period from 1969 to 1996, 209 consecutive surgically resected cases of carcinoma of the ampulla of Vater and 10 cases of adenoma were placed on file in the Department of Anatomic Pathology, Pathological Sciences, Graduate School of Medical Sciences, Kyushu University. The criteria for these tumors of the ampulla of Vater was as follows: the tumors were derived from the duodenal mucosa (only the ampullary region), the common channel, the intraduodenal pancreatic duct, or the intraduodenal common bile duct; this was confirmed by $5-\mathrm{mm}$ stepwise tissue sections from the specimens. These histologic sections, cut from $10 \%$ formalin-fixed, paraffin blocks, were routinely stained with hematoxylin and eosin. Macroscopically, the ampullary carcinomas were categorized into three groups according to those designated by
Tasaka et al. and Yamaguchi and Enjoji $(2,23)$ : intramural protruding, exposed protruding, and ulcerating form (Figs. 1 to 3 ). Microscopically, the tumors were classified by the system in the World Health Organization (WHO) monograph (24) as papillary adenocarcinoma, adenocarcinoma (including well differentiated, moderately differentiated and poorly differentiated), mucinous adenocarcinoma, signet-ring cell carcinoma, clear cell adenocarcinoma, adenosquamous carcinoma, squamous carcinoma, small cell carcinoma, and undifferentiated carcinoma. We classified the degree of dysplasia of the lesions adjacent to the carcinoma of the ampulla of Vater using the criteria of Baczako et al. (6). We were able to classify three degrees of dysplasia: mild, moderate, or severe. The degree of dysplasia was assessed by structural or cellular atypia: degrees of pseudostratification, nuclear atypia, loss of polarity, and occasional mitotic figures. Mild dysplasia was characterized by elongated nuclei in tall columnar epithelial cells and mild nuclear hyperchromasia. Moderate dysplasia was defined as the presence of pseudostratification with moderate intraglandular budding and bridging, accompanied by certain polymorphic nuclear changes and moderate hyperchromasia. The term severe dysplasia was used if the epithelium showed widening and marked pseudostratification, with cuboidal cells as well as marked budding and bridging. The nuclei were hyperchromatic, vacuolated, and polymorphic. Mitotic figures were frequently detected in the areas lacking invasive growth. We used TNM classification for staging ampullary carcinomas (24) as follows: Stage I, the tumor cells are confined to the ampullary region; Stage II, the tumor cells are restricted to the duodenal wall; Stages III and IV, the tumor invasion is limited to $2 \mathrm{~cm}$ or over in the pancreas. If the regional lymph node

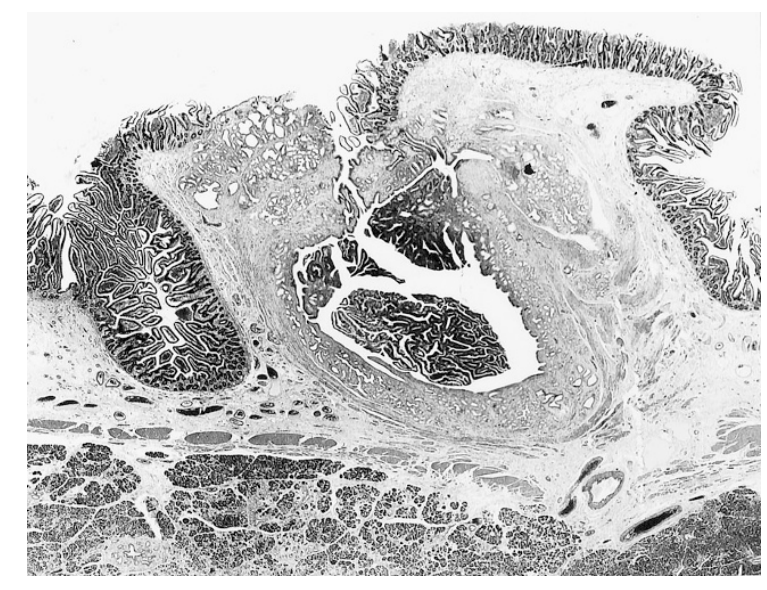

FIGURE 1. Intramural protruding form. The tumor cells proliferate in a papillary fashion and are restricted to within the muscle of Oddi. We define this tumor as an early ampullary carcinoma (hematoxylin and eosin staining, $10 \times$ ). 


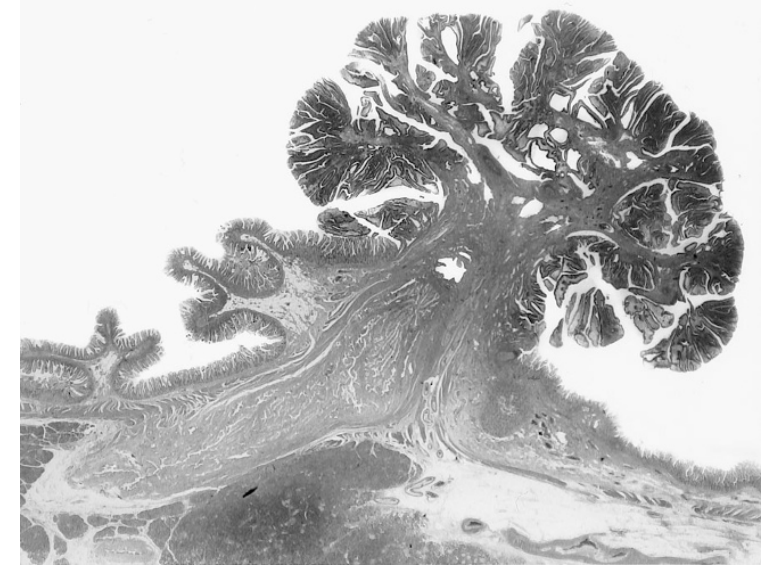

FIGURE 2. Exposed protruding form. Papillary proliferation of the tumor cells in the common channel extends to the surface of the ampulla of Vater (hematoxylin and eosin staining, $3 \times$ ). This case is also included in the category of early ampullary carcinoma.

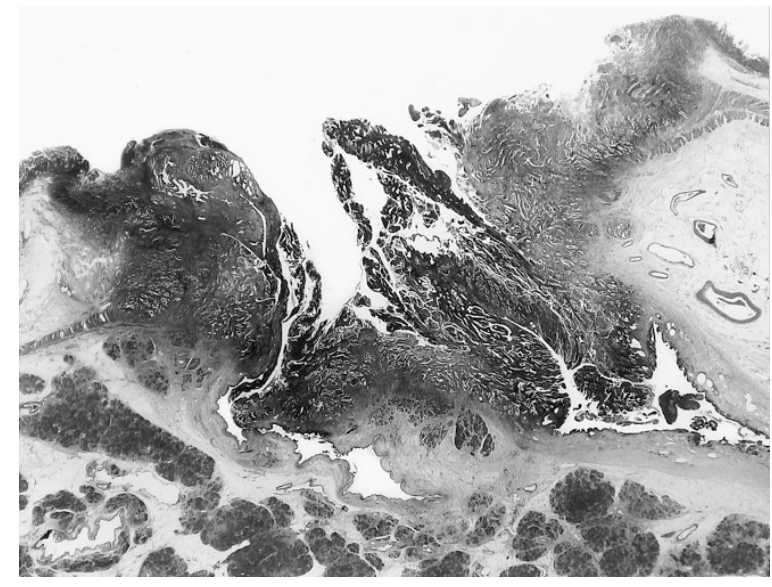

FIGURE 3. Ulcerating form. The carcinoma cells have invaded the duodenal wall and pancreatic parenchyma, indicating advanced ampullary carcinoma (hematoxylin and eosin staining, $3 \times$ ).

metastasis is present, the level goes up from Stage I or II to Stage III. In line with the procedure of studies elsewhere $(2,25)$, we defined early ampullary carcinoma as a tumor that was restricted to within the muscle of Oddi and defined advanced ampullary carcinoma as a tumor extending beyond the muscle of Oddi. Other clinicopathological factors examined were as follows: degree of invasion of the surrounding tissue, such as duodenal wall; pancreatic parenchyma; the presence or absence of lymphatic permeation; venous invasion; perineural invasion; and the presence of regional lymph node metastasis.

From 219 specimens, 146 specimens of carcinoma and 9 specimens of pure adenoma were available for immunohistochemical staining in this study. An immunohistochemical study was performed on the formalin-fixed, paraffin-embedded sections by the streptavidin-biotin peroxidase-complex method using a Histofine SAB-PO kit (Nishirei, Tokyo, Japan), with monoclonal antibodies specific for p53 $(1 ; 100$,
Pab1801, Oncogene Science, Uniondale, NY) and Ki-67 (1:100, MIB-1, Immunotech, Marseille, France). After deparaffinization, the endogenous peroxidase activity was blocked with hydration and hydrogen peroxidase. The sections for p53 were pretreated by heating in a microwave oven at $100^{\circ} \mathrm{C}$ in phosphate buffered saline for 20 minutes, whereas the sections for Ki-67 were boiled in $\mathrm{pH} 6.0$ citrate buffer for 30 minutes. The primary antibodies were applied to tissue overnight at $4^{\circ} \mathrm{C}$ before being incubated for 20 minutes with a secondary biotinylated antibody, followed by further incubation with a streptavidinbiotin-peroxidase complex reagent for 20 minutes. The sections were thoroughly washed with phosphate buffered saline between steps. The sites of peroxidase were visualized with $3,3^{\prime}$-diaminobenzidine tetrahydrochloride. Hematoxylin was used for counterstaining. Appropriate positive and negative controls were included.

The extent of the nuclear reactivity for p53 was classified into four categories: -, no expression; \pm , low expression (1 to $10 \%$ immunoreactive tumor cells); +, moderate expression (10 to 50\%); and ++ , high expression (more than 50\%). The latter two $(+,++)$ were regarded as significantly positive in this study (Fig. 4, e-f).

In the case of Ki-67 antigen staining, the number of positively stained nuclei out of 1000 cancer cell nuclei was counted and expressed as a percentage in the form of a labeling index (Fig. 4g).

Immunohistochemical analysis was performed without access to any of the clinical information about each specimen. With regard to the carcinoma with adenomatous areas (CWA), adenomatous and carcinomatous areas were separately evaluated.

Complete follow-up information was available for 198 patients with carcinoma and 10 patients with pure adenoma. The relationship between each of the clinicopathological factors or the expression of each of the oncogenes and the histological groups was assessed by $\chi^{2}$ test or Fisher's exact test, whereas differences in the Ki-67 labeling index between the groups were analyzed by the Mann-Whitney or Kruskal-Wallis test. Survival curves were estimated by the Kaplan-Meier method, and differences between the curves were measured using the log rank test. All variables that were significant at the $5 \%$ level in the univariate or multivariate analysis were included in Cox's proportional hazards regression model. Model selection for identifying the subset of significant variables was based on a forward stepwise procedure using the maximum likelihood ratio test.

\section{RESULTS}

\section{Sex and Age}

The 198 patients with carcinoma of the ampulla of Vater were all Japanese, ranging in age from 33 to 


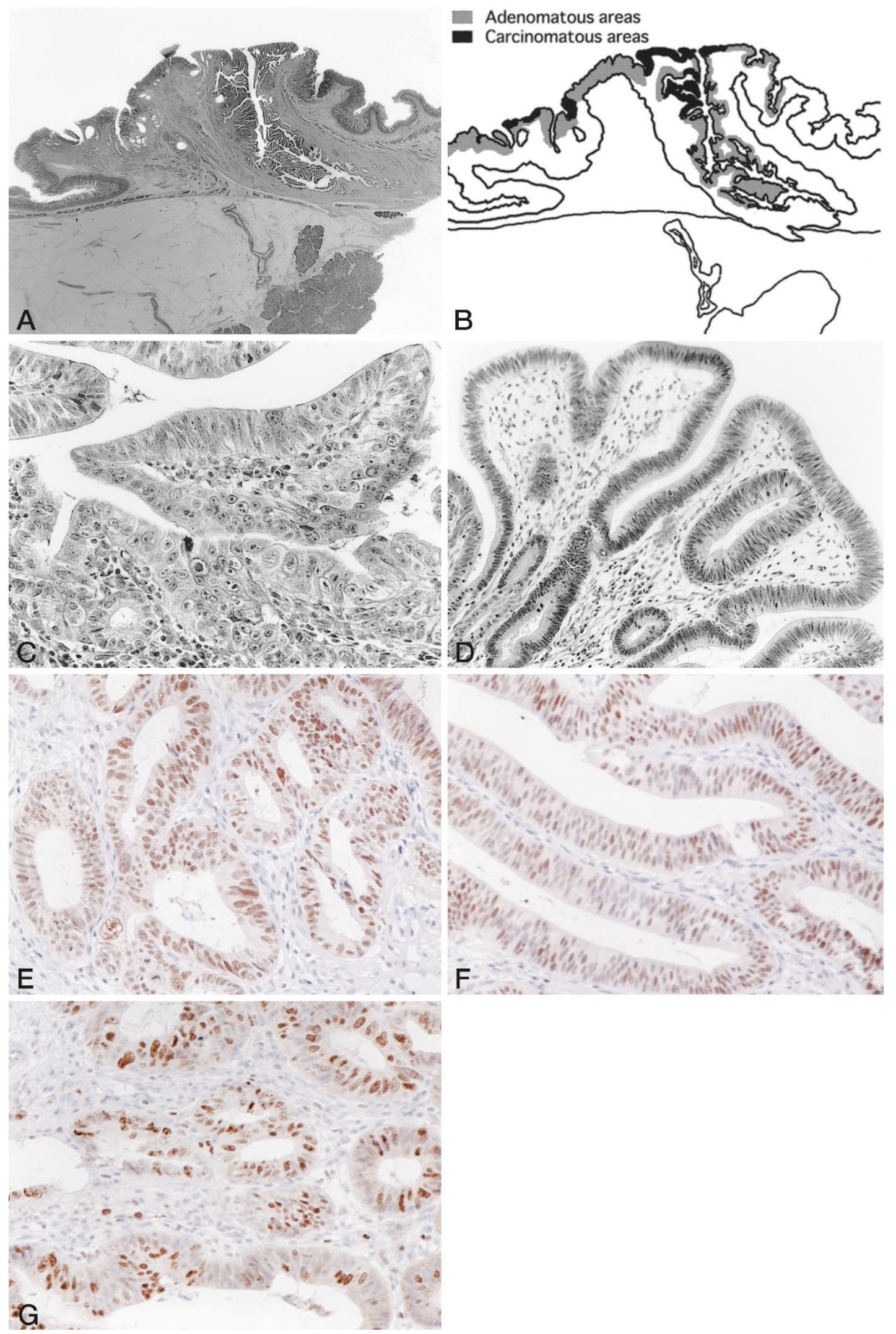

FIGURE 4. a, Exposed protruding form of ampullary carcinoma. The papillary tumor proliferates in the common channel, on the surface of duodenal papilla, and within the intraduodenal common bile duct (hematoxylin and eosin staining, $3 \times$ ). b. Distribution of carcinomatous and adenomatous areas: the adenomatous areas are mostly present within the rim of the tumor and in the deep portion of the common channel and the common bile duct. c, Carcinomatous areas: tumor cells having bizarre nuclei and prominent nucleoli exhibit irregular tubules (hematoxylin and eosin staining, $160 \times$ ). d, Adenomatous areas: the epithelium proliferates in a tubular or villous fashion, and epithelial cells have moderate to severe nuclear atypia (hematoxylin and eosin staining, 120×). e, p53 immunohistochemical stain in carcinomatous areas: in this case, carcinoma cells exhibit diffusely positive reaction in their nuclei $(2+; 120 \times)$. f, p53 immunohistochemical stain in adenomatous areas: tumor cells are diffusely positive $(2+; 160 \times) . \mathbf{g}, 60.5 \%$ of the tumor cells are positive for Ki-67 $(120 \times)$. 
81 years (mean, $60.5 \mathrm{y}$ ). There were 102 men and 96 women (Table 1). The 10 patients with pure adenoma included 7 men and 3 women, ranging in age from 48 to 72 years (mean, $60 \mathrm{y}$ ).

\section{Macroscopic Appearances}

The correlation between macroscopic type and clinicopathologic findings of carcinoma of the ampulla of Vater is summarized in Table 1. Macroscopically, 32 of the cases were of an intramural protruding form, whereas 97 cases and 69 cases were of an exposed protruding form and an ulcerating form, respectively. The ulcerating tumors were the largest, averaging $2.5 \mathrm{~cm}$ in the greater diameter, but the intramural protruding tumors were the smallest, averaging $1.7 \mathrm{~cm}$. The average diameter of the 10 pure adenomas was $2.4 \mathrm{~cm}$.

\section{Histopathologic Findings}

Of the 198 carcinomas, 33 were papillary adenocarcinomas, 157 were adenocarcinomas (104 well differentiated, 39 moderately differentiated, and 14 poorly differentiated), 2 were adenosquamous carcinomas, and 6 were mucinous adenocarcinomas (Table 1). In 83 of the 198 carcinoma cases $(42 \%)$, there were adenomatous areas surrounding the carcinoma: these cases included 13 papillary adenocarcinomas; 54 well-differentiated, 12 moderately differentiated, and 3 poorly differentiated adenocarcinomas; and 1 mucinous adenocarcinoma. There were 19 early and 179 advanced carcinomas.
The degree of dysplastic epithelium within the adenomatous areas in all the cases of ampullary carcinoma exhibited almost moderate dysplasia and focally mild dysplasia. Ten pure adenomas were composed of mild to moderate dysplastic epithelium. In the two groups, early carcinoma (TNM classified as Stage I) and advanced carcinoma (Stages II to IV), cases with adenomatous areas numbered 13 and 70, respectively.

\section{Immunohistochemical Study}

One hundred forty-six specimens of carcinoma and nine specimens of pure adenoma were available for immunohistochemical staining with p53 and Ki-67 monoclonal antibodies. The immunohistochemical staining patterns for p53 and Ki-67 are summarized in Tables 3 and 4. Eighty-six of 146 cases with carcinoma exhibited an overexpression of p53. The percentage of cases showing p53 overexpression was $91.7 \%(11 / 12)$ for early carcinomas and $55.9 \%(75 / 134)$ for advanced carcinomas. Moreover, $0 \%$ in pure adenomas, $36 \%$ in adenomatous areas of CWA and $62 \%$ in carcinomatous areas of CWA, and $56 \%$ in pure carcinoma were positive for p53 immunohistochemical staining.

With regard to Ki-67, the labeling index was 31.3 on average, ranging from 0 to 72.4 . However, no significant difference was found between accumulation of p53 protein or the Ki-67 labeling index and other clinicopathological factors.

TABLE 1. Prognostic Factors in 198 Patients with Carcinoma of the Ampulla of Vater

\begin{tabular}{|c|c|c|c|c|c|c|c|c|c|}
\hline & & \multirow{2}{*}{$n(\%)$} & \multicolumn{2}{|c|}{ Size $(\mathrm{cm})$} & \multicolumn{2}{|c|}{ Age } & \multirow{2}{*}{$\begin{array}{l}\text { Adenomatous } \\
\text { Area }(+)(\%)\end{array}$} & \multirow{2}{*}{\multicolumn{2}{|c|}{ 5-Year Survival Rate (\%) }} \\
\hline & & & Average & Range & Average & Range & & & \\
\hline & Total & $198(100)$ & 2.3 & $0.4-7.3$ & 60.5 & $33-81$ & 42 & 42.0 & \\
\hline \multirow[t]{2}{*}{ Sex } & M & $102(51.5)$ & 2.4 & $0.6-7.3$ & 59.4 & $33-80$ & 45 & 8.40 & \\
\hline & $\mathrm{F}$ & $96(48.5)$ & 2.2 & $0.4-5.2$ & 61.6 & $38-81$ & 39 & 45.8 & \\
\hline \multirow[t]{3}{*}{$\begin{array}{l}\text { Macroscopic } \\
\text { findings }\end{array}$} & $\begin{array}{l}\text { Intramural } \\
\text { protruding } \\
\text { form }\end{array}$ & $32(16.2)$ & 1.7 & $0.4-3.5$ & & & 41 & 44.3 & \\
\hline & $\begin{array}{l}\text { Exposed } \\
\text { protruding } \\
\text { form }\end{array}$ & $97(49)$ & 2.3 & $0.7-5.2$ & & & 56 & 46.4 & \\
\hline & Ulcerating form & $69(34.8)$ & 2.5 & $0.9-7.3$ & & & 23 & 36.1 & \\
\hline \multirow[t]{7}{*}{$\begin{array}{l}\text { Microscopic } \\
\text { findings }\end{array}$} & $\begin{array}{l}\text { Well differentiated } \\
\text { group }\end{array}$ & $137(69.2)$ & 2.4 & $0.4-7.3$ & & & 49 & 47.7 & \multirow{7}{*}{$P=0.0084^{*}$} \\
\hline & Papillary & $33(16.7)$ & 2.5 & $1.1-5.0$ & & & 39 & 54.3 & \\
\hline & Well & $104(52.5)$ & 2.3 & $0.4-7.3$ & & & 52 & 45.9 & \\
\hline & Moderately & $39(19.7)$ & 2 & $0.7-5.6$ & & & 31 & 30.0 & \\
\hline & Poorly & $14(7.1)$ & 2.1 & $1.1-3.4$ & & & 24 & 21.4 & \\
\hline & Adenosquamous & $2(1)$ & 2.5 & $1.5-3.5$ & & & 0 & 50.0 & \\
\hline & Mucinous & $6(3)$ & 2.5 & $1.3-4.3$ & & & 17 & 33.3 & \\
\hline \multirow[t]{5}{*}{ TNM Stage } & Early I & $19(9.6)$ & 2 & $0.4-4.1$ & 57 & $33-75$ & 68.4 & 81.4 & \multirow{5}{*}{$\begin{array}{l}P=0.128^{*} \\
P=0.0023^{*}\end{array}$} \\
\hline & Advanced & 179 & & & & & 39.1 & 38.6 & \\
\hline & II & 79 (39.9) & 2.1 & $0.6-5$ & 63 & $39-81$ & 46.8 & 47.6 & \\
\hline & III & $95(50)$ & 2.3 & $0.5-5.6$ & 59 & $38-80$ & 34.7 & 33.4 & \\
\hline & IV & $5(2.5)$ & 4.9 & $2.7-7.3$ & 58 & $47-63$ & 60 & 0.0 & \\
\hline
\end{tabular}

${ }^{*}$ Logrank test. 
TABLE 2. Prognostic Factors in 198 Patients with Carcinoma of the Ampulla of Vater

\begin{tabular}{|c|c|c|c|c|}
\hline & & $n$ & 5 Year Survival Rate (\%) & $P$ value* \\
\hline \multirow[t]{2}{*}{ Invasion of the pancreas } & - & 83 & 59.2 & \multirow[b]{2}{*}{$P<0.0001$} \\
\hline & + & 115 & 30.3 & \\
\hline \multirow[t]{2}{*}{ Invasion of the duodenal wall } & - & 86 & 58.5 & \multirow[b]{2}{*}{$P<0.0001$} \\
\hline & + & 112 & 29.6 & \\
\hline \multirow[t]{2}{*}{ Lymphatic permeation } & - & 129 & 51.3 & \multirow[b]{2}{*}{$P=0.0002$} \\
\hline & + & 69 & 25.1 & \\
\hline \multirow[t]{2}{*}{ Venous invasion } & - & 86 & 58.2 & \multirow[b]{2}{*}{$P=0.0003$} \\
\hline & + & 112 & 30.4 & \\
\hline \multirow[t]{3}{*}{ Perineural invasion } & - & 154 & 47.4 & \multirow[b]{2}{*}{$P=0.0034$} \\
\hline & + & 44 & 23.8 & \\
\hline & Total & 198 & & \multirow{4}{*}{$P=0.0047$} \\
\hline \multirow[t]{3}{*}{ Regional lymph node metastasis } & - & 97 & 53.4 & \\
\hline & + & 92 & 30.1 & \\
\hline & Total & 189 & & \\
\hline
\end{tabular}

* Logrank test.

TABLE 3. p53 Immunohistochemical Staining for Adenoma and Carcinoma with/without Adenomatous Areas

\begin{tabular}{|c|c|c|}
\hline & & $\begin{array}{c}\text { p53 Immunohistochemical } \\
\text { Staining }(+)\end{array}$ \\
\hline Pure adenoma & $(n=9)$ & 0 \\
\hline \multirow[t]{2}{*}{ Carcinoma with adenomatous areas (CWA) } & $(n=61)$ & $22(36 \%)$ \\
\hline & Carcinomatous area & $38(62 \%)$ \\
\hline Carcinoma without adenomatous areas (pure carcinoma) & $(n=85)$ & $48(56 \%)$ \\
\hline Total & & $86(58.9 \%)$ \\
\hline
\end{tabular}

TABLE 4. Clinicopathological Factors and Immunohistochemical Staining in TNM Stage of the Ampulla of Vater

\begin{tabular}{|c|c|c|c|c|c|}
\hline \multirow[b]{2}{*}{$\begin{array}{c}\text { TNM } \\
n\end{array}$} & \multirow[b]{2}{*}{$\begin{array}{c}\text { Total } \\
146\end{array}$} & \multirow{2}{*}{$\begin{array}{c}\text { Early } \\
\text { Stage I } \\
12\end{array}$} & \multicolumn{3}{|c|}{ Advanced } \\
\hline & & & $\begin{array}{c}\text { Stage II } \\
61\end{array}$ & $\begin{array}{l}\text { Stage III } \\
68\end{array}$ & $\begin{array}{c}\text { Stage IV } \\
5\end{array}$ \\
\hline Age (range) & $61(38-81)$ & $61(43-75)$ & $64(39-81)$ & $58(38-79)$ & $58(47-63)$ \\
\hline Sex $(M: F)$ & 73:73 & $7: 5$ & $25: 36$ & $38: 30$ & $3: 2$ \\
\hline Macroscopic findings (IP:EP:U) & 18:75:53 & 3:9:0 & 6:38:17 & 9:27:32 & $0: 1: 4$ \\
\hline Microscopic findings (Well:Mod:Por:Muc:Adsq) & 98:32:9:6:1 & 12:0:0:0:0 & $43: 11: 5: 2: 0$ & $38: 21: 4: 4: 1$ & 5:0:0:0:0 \\
\hline Including adenomatous areas (\%) & 41.8 & 75 & 42.6 & 29.4 & 60 \\
\hline Ki-67 labeling index (mean) (\%) & 31.3 & 32.4 & 30.9 & 31.3 & 31.1 \\
\hline p53(+) (\%) & 58.9 & 91.7 & 55.7 & 57.4 & 40 \\
\hline 5 -year survival rate $(\%)^{*}$ & 39.5 & 83.3 & 43.7 & 33.1 & 0 \\
\hline
\end{tabular}

IP, intramural protruding form; EP, exposed protruding form; U, ulcerating form; Mod, moderately differentiated; Por, poorly differentiated; Muc, mucinous; Adsc, adenosquamons.

* Logrank test $P=0.0226$.

\section{Survival and Prognostic Factors}

Follow-up times ranged from 1 to 298 months (median follow-up, 32 months). The overall postoperative survival rate was $42 \%$ after 5 years (Table 1). Tasaka et al. (23) had reasoned that the morphological findings were related with tumor progression because during the early phase, the tumor assumed a protuberant form, whereas by the advanced phase, the tumor had transformed into an ulcerating form; however, there was no significant difference in prognosis between the two macroscopic forms. Size and sex had no great influence on survival. The survival rates in the cases of papillary carcinoma; well-differentiated, moderately differentiated, and poorly differentiated carcinoma; adenosquamous carcinoma; and mucinous carcinoma were $54.3,45.9,30,21.4,50$, and $33.3 \%$, respectively. Furthermore, there were statistically significant differences in the survival curves among the patients in the well-differentiated carcinoma group (papillary and well-differentiated carcinoma), those in the moderately differentiated group, and those in the poorly differentiated group (Table 1). Clinicopathology also showed a significant difference (Table 2). Moreover, there was a significant difference in the survival probability between the group of carcinomas restricted to within the muscle of Oddi (i.e., early carcinoma) and the group of carcinomas extending beyond the muscle (advanced carcinoma) (Table 1). Accumulation of p53 protein and the Ki-67 labeling index demonstrated no statistically significant difference in prognosis.

A multivariate analysis using Cox's proportional hazards model evaluated the influence of the each clinicopathologic variables on prognosis (Table 5). Strong predictors for a better prognosis were noted in cases without any lymphatic permeation, invasion of the pancreas, or perineural invasion. 


\begin{tabular}{lccc}
\hline \multicolumn{1}{c}{ Variable } & $\begin{array}{c}\text { Estimated } \\
\text { Coefficient }\end{array}$ & Standard Error & Relative Risk \\
\hline Lymphatic permeation $(+/-)$ & 0.3001 & 0.1086 & 1.35 \\
Invasion of the pancreas $(+/-)$ & 0.3017 & 0.121 & 1.3522 \\
Perineural invasion $(+/-)$ & 1.2765 & 0.4786 & 3.5839 \\
\hline
\end{tabular}

\section{DISCUSSION}

In the current study, long-term survival was found to be correlated independently with the histological classification and with the degree of invasion of the pancreas or duodenal wall, lymphatic permeation, venous invasion, perineural invasion, and regional lymph node metastasis. These results were similar to those of studies published elsewhere $(2,18)$. Strong predictors for a better prognosis were noted in those cases without any lymphatic permeation or invasion of the pancreas in multivariate analysis.

In previous studies, adenomatous areas were found frequently within or in the vicinity of carcinoma of the ampulla of Vater (30-91\%; 8, 9, 10, 25). Moreover, areas with severe dysplasia and/or carcinoma in situ were often seen in those tubular adenomas that showed moderate dysplasia $(2,26)$. Among our 198 cases, we found 83 (42\%) carcinomas of the ampulla of Vater that contained adenomatous areas. There was no significant difference in prognosis between CWAs and carcinomas without adenomatous areas. Carcinoma of the ampulla of Vater showed a tendency to contain adenomatous areas far more frequently during the early phase (70\%) than the advanced phase (38\%). On the basis of these facts, we concluded that adenomatous areas had been present at the onset of the tumors but that the rate at which the tumors contained adenomatous areas decreased as the tumors advanced. In previous studies, the basic evidence for this was the coexistence of adenoma and carcinoma within the same lesion. Some authors have presented the hypothesis that carcinomas arise within adenomas in the ampulla of Vater, similar to the case with the adenoma-carcinoma development hypothesis of colorectal carcinoma $(2,13)$. Recently, a genetic alteration model during colorectal tumor development has attracted much interest, leading to several studies $(11,12)$. In our study, positivity of p53 immunohistochemical staining was $0 \%$ in pure adenomas, $36 \%$ in adenomatous areas of CWA and $62 \%$ in carcinomatous areas of CWA, and $56 \%$ in pure carcinoma. This result is consistent with the adenoma-carcinoma development hypothesis.

Scarpa et al. (13) reported that p53 gene mutation was detected in 8/11 carcinomas of the ampulla of Vater using the PCR-SSCP (single-strand conformation polymorphism) method, and 7 of those 11 carcinomas exhibited positivity for p53 immunohisto- chemically, but there was no positive stain for p53 protein within the adenomatous areas of those cases of carcinoma that had such areas. Diamantis et al. (15) observed significantly higher p53 positivity in high-grade neoplasms compared with low-grade ones. Moreover, Ajiki et al. (27) reported similar results for gallbladder neoplasms in that the tumor cells of adenoma and dysplasia were negative for p53 staining, whereas carcinoma cells were positive. On the other hand, Younes et al. (16) reported that $40 \%$ of adenomas and $94 \%$ of carcinomas of the ampulla of Vater were positive for p53. In our study, we revealed positive p53 staining present in none of nine pure adenomas but present in 22 of 38 adenomatous areas of CWAs (58\%; Table 3). On the basis of all the above reports, we deduced that the incidence of accumulation of p53 gene alterations was smaller in pure adenoma with mild to moderate dysplasia than in adenomatous area of CWA with moderate dysplasia. Fiftynine percent of all 146 cases of carcinoma of the ampulla of Vater demonstrated p53 positivity, this being comparatively lower than in studies elsewhere (64 to $94 \% ; 13-16$ ). There was no significant difference in long-term survival between the p53-positive group and the p53-negative group (5-y survival rates, 33 and $48.2 \%$, respectively). p53 positivity of pure adenomas, early carcinomas, and advanced carcinomas was 0 , 92.3, and $55.6 \%$, respectively. The current results, showing that p53 positivity in the advanced cancer group was lower than that in the early cancer group, could be due to the additional allele loss on the remaining side of Chromosome 17 or to a new mutation that stops the prolongation of the half-life of p53 protein, but there is no conclusive evidence for this.

In conclusion, p53 immunohistochemical staining may be useful in the diagnosis of carcinoma of the ampulla of Vater if the submitted specimen contains adenomatous areas. It is most likely that the molecular events leading to p53 accumulation in neoplasms of the ampulla of Vater occurred relatively late during the oncogenetic process. These results did not contradict the adenoma-carcinoma hypothesis. However, further molecular investigations are necessary to verify whether this is accurate.

Acknowledgments: The authors thank Naoko Kinukawa for her aid with the multivariate analysis and Miss K. Miller (Royal English Language Center, Fukuoka, Japan) for revising the English used in this manuscript. 


\section{REFERENCES}

1. Nakase A, Matsumoto Y, Uchida K, Honjo I. Surgical treatment of cancer of the pancreas and the periampullary region: cumulative results in 57 institutions in Japan. Ann Surg 1977;185:52-7.

2. Yamaguchi K, Enjoji M. Carcinoma of the ampulla of Vater. A clinicopathologic study and pathologic staging of 109 cases of carcinoma and 5 cases of adenoma. Cancer 1987;59:50615 .

3. Shutze WP, Sack J, Aldrete JS. Long-term follow-up of 24 patients undergoing radical resection for ampullary carcinoma, 1953 to 1988. Cancer 1990;66:1717-20.

4. Klöppel G, Maillet B. Histological typing of pancreas and periampullary carcinoma. Eur J Surg Oncol 1991;17:13952.

5. Nakao A, Harada A, Nonami T, Kishimoto W, Takeda S, Ito K, et al. Prognosis of cancer of the duodenal papilla of Vater in relation to clinicopathological tumor extension. Hepatogastroenterology 1994;41:73-8.

6. Baczako K, Büchler M, Beger HG, Kirkpatrick CJ, Haferkamp O. Morphogenesis and possible precursor lesions of invasive carcinoma of the papilla of Vater: epithelial dysplasia and adenoma. Hum Pathol 1985;16:305-10.

7. Perzin KH, Bridge MF. Adenomas of the small intestine: a clinicopathologic review of 51 cases and a study of their relationship to carcinoma. Cancer 1981;48:799-819.

8. Kozuka S, Tsubone M, Yamaguchi A, Hachisuka K. Adenomatous residue in cancerous papilla of Vater. Gut 1981;22: 1031-4.

9. Seifert E, Schulte F, Stolte M. Adenoma and carcinoma of the duodenum and papilla of Vater: a clinicopathologic study. Am J Gastroenterol 1992;87:37-42.

10. Talbot IC, Neoptolemos JP, Shaw DE, Carr-Locke D. The histopathology and staging of carcinoma of the ampulla of Vater. Histopathology 1988;12:155-65.

11. Vogelstein B, Fearon ER, Hamilton SR, Kern SE, Preisinger AC, Leppert M, et al. Genetic alterations during colorectal tumor development. N Engl J Med 1988;319:525-32.

12. Fearon ER, Vogelstein B. A genetic model for colorectal tumorigenesis. Cell 1990;61:759-67.

13. Scarpa A, Capelli P, Zamboni G, Oda T, Mukai K, Bonetti F, et al. Neoplasia of the ampulla of Vater; Ki-ras and p53 mutations. Am J Pathol 1993;142:1163-72.

14. Teh M, Wee A, Path MRC, Raju GC. An immunohistochemical study of p53 protein in gallbladder and extrahepatic bile duct/ampullary carcinomas. Cancer 1994;74:1542-5.

15. Diamantis I, Karamitopoulou E, Perentes E, Zimmermann A. p53 protein immunoreactivity in extrahepatic bile duct and gallbladder cancer: correlation with tumor grade and survival. Hepatology 1995;22:774-9.

16. Younes M, Riley S, Genta RM, Mosharaf M, Mody DR. p53 protein accumulation in tumors of the ampulla of Vater. Cancer 1995;76:1150-4.

17. Zhu L, Kim K, Domenico DR, Appert HE, Howard JM. Adenocarcinoma of duodenum and ampulla of Vater: clinicopathology study and expression of p53, c-neu, TGF-alpha, CEA, and EMA. J Surg Oncol 1996;61:100-5.

18. Dorandeu A, Raoul JL, Siriser F, Rioux NL, Gosselin M, Martin ED, et al. Carcinoma of the ampulla of Vater: prognostic factors after curative surgery: a series of 45 cases. Gut 1997; 40:350-5.

19. Dierendonck JH, Wijsman JH, Keijzer R, Velde CJH, Cornelisse CJ. Cell-cycle-related staining patterns of antiproliferating cell nuclear antigen monoclonal antibodies: comparison with BrdUrd labeling and Ki-67 staining. Am J Pathol 1991;138:1165-72.

20. Dervan PA, Magee HM, Buckley C, Carney DN. Proliferating cell nuclear antigen counts in formalin-fixed paraffinembedded tissue correlate with Ki-67 in fresh tissue. Am J Clin Pathol 1992(5 Suppl);97:S21-8.

21. Schmitt FC, Ferreira MP. MIB-1 is a suitable marker of proliferative activity in formalin-fixed, paraffin-embedded sections of breast cancer. Int J Surg Pathol 1995;2:287-94.

22. Gee JMW, Douglas-Jones A, Hepburn P, Sharma AK, McClelland RA, Ellis IO, et al. A cautionary note regarding the application of Ki-67 antibodies to paraffin-embedded breast cancers. J Pathol 1995;177:285-93.

23. Tasaka K, Watanabe H, Enjoji M. Carcinoma of the pancreas, gallbladder, extrahepatic bile ducts and duodenal papilla: a statistical observation of 137 autopsy cases [abstract]. Fukuoka Igaku Zasshi 1975;66:486-99.

24. Albores-Saavedra J, Henson DE, Sobin LH. Histological typing of tumours of the gallbladder and extrahepatic bile ducts. Geneva, Switzerland: World Health Organization; 1991.

25. Neoptolemos JP, Talbot IC, Shaw DC, Carr-Locke DL. Longterm survival after resection of ampullary carcinoma is associated independently with tumor grade and a new staging classification that assesses local invasiveness. Cancer 1988; 61:1403-7.

26. Yamagichi K, Enjoji M. Adenoma of the ampulla of Vater: putative precancerous lesion. Gut 1991;32:1558-61.

27. Ajiki T, Onoyama H, Yamamoto M, Asaka K, Fujimori T, Maeda S, et al. p53 protein expression and prognosis in gallbladder carcinoma and premalignant lesions. Hepatogastroenterology 1996;43:521-6. 